\title{
Dual exponential polynomials and a problem of Ozawa
}

\author{
J. Heittokangas, K. Ishizaki, K. Tohge and Z.-T. Wen
}

\begin{abstract}
Complex linear differential equations with entire coefficients are studied in the situation where one of the coefficients is an exponential polynomial and dominates the growth of all the other coefficients. If such an equation has an exponential polynomial solution $f$, then the order of $f$ and of the dominant coefficient are equal, and the two functions possess a certain duality property. The results presented in this paper improve earlier results by some of the present authors, and the paper adjoins with two open problems.
\end{abstract}

Key words: Dual exponential polynomials, exponential sum, finite order, linear differential equation, Ozawa's problem, value distribution.

2020 MSC: Primary 30D15; Secondary 30D35.

\section{Introduction}

Frei 2] has proved that the differential equation

$$
f^{\prime \prime}+e^{-z} f^{\prime}+\alpha f=0, \quad \alpha \in \mathbb{C} \backslash\{0\},
$$

has a subnormal (that is, non-trivial and finite-order) solution if and only if $\alpha=-m^{2}$ for a positive integer $m$. The subnormal solution $f$ is a polynomial in $e^{z}$ of degree $m$, that is, an exponential sum of the form

$$
f(z)=1+C_{1} e^{z}+\cdots+C_{m} e^{m z}, \quad C_{j} \in \mathbb{C} .
$$

It was discovered in [18, Lemma 1] that in this representation one has $C_{j} \neq 0$ for $1 \leq j \leq m$. Substituting the subnormal solution $f$ into (1.1), we get

$$
\sum_{j=1}^{m} C_{j} j^{2} e^{j z}+\sum_{j=1}^{m} C_{j} j e^{(j-1) z}-m^{2} \sum_{j=1}^{m} C_{j} e^{j z}=m^{2} .
$$

By the Borel-Nevanlinna theorem [3, pp. 70, 108], or simply by an elementary observation on three polynomials in $e^{z}$, this gives rise to the recursive formula

$$
C_{1}=m^{2}, \quad\left(m^{2}-j^{2}\right) C_{j}=(j+1) C_{j+1}, \quad 1 \leq j \leq m,
$$

from which $C_{j}=\frac{1}{j !} \prod_{k=0}^{j-1}\left(m^{2}-k^{2}\right)$ for $1 \leq j \leq m$. Due to the presence of the transcendental coefficient $e^{-z}$, any solution of (1.1) linearly independent with $f$ in (1.2) must be of infinite order [7]. For example, when $\alpha=-1$, the function $g(z)=\exp \left(e^{-z}+z\right)$ is an infinite order solution of (1.1) and linearly independent with $f(z)=1+e^{z}$. 
Ozawa [15] showed that if $a \neq 0$, then the non-trivial solutions of

$$
f^{\prime \prime}+e^{-z} f^{\prime}+(a z+b) f=0
$$

are of infinite order of growth. If $P(z)$ is a non-constant polynomial, the question whether all non-trivial solutions of

$$
f^{\prime \prime}+e^{-z} f^{\prime}+P(z) f=0
$$

are of infinite order of growth has been known as the Ozawa problem. This problem has been answered affirmatively for particular polynomials $P(z)$ by Amemiya-Ozawa [1] and by Gundersen [4], while the complete solution is by Langley [12].

We proceed to state three new examples of Frei-Ozawa type.

Example 1.1 If $H$ is an arbitrary entire function, then $f(z)=e^{z}+1$ solves

$$
f^{\prime \prime}+\left(H-1+H e^{-z}\right) f^{\prime}-H f=0 .
$$

Of particular interest is the case when $H$ is a polynomial.

Example 1.2 The function $f(z)=1+(1-3 c)\left(e^{z}+\left(1-\frac{3}{4} c\right)\right) e^{2 z}, c \in \mathbb{C} \backslash\left\{\frac{1}{3}, \frac{4}{3}\right\}$, with two exponential terms solves

$$
f^{\prime \prime}+\left(-\frac{5}{3}-c+\frac{2}{3} e^{-z}\right) f^{\prime}+\left(2 c-\frac{2}{3}\right) f=0 .
$$

Example 1.3 The function $f(z)=1+3 e^{2 z}+\sqrt{6} i e^{3 z}$ with two exponential terms solves

$$
f^{\prime \prime}+\left(1-\sqrt{6} i e^{-z}+2 e^{-2 z}\right) f^{\prime}-12 f=0
$$

where the transcendental coefficient has two exponential terms also. By making a change of variable $z \rightarrow w z$, where $w \in \mathbb{C} \backslash\{0\}$, we see that

$$
g^{\prime \prime}+w\left(1-\sqrt{6} i e^{-w z}+2 e^{-2 w z}\right) g^{\prime}-12 w^{2} g=0
$$

has a solution $g(z)=1+3 e^{2 w z}+\sqrt{6} i e^{3 w z}=f(w z)$.

One might wonder about possible examples of solutions $f$ with a single exponential term and of transcendental coefficients $A(z)$ having at least two exponential terms. The non-existence of such examples will be confirmed in Theorem 3.2 below. For example, it will be shown that a function $f(z)=1+b e^{w z}$ for $b, w \in \mathbb{C}$ is a solution of

$$
f^{\prime \prime}+\left\{P_{1}(z)+P_{2}(z) e^{-w z}\right\} f^{\prime}-P(z) f=0
$$

for $P(z), P_{1}(z), P_{2}(z) \in \mathbb{C}[z]$ if and only if $P_{1}(z)=\frac{1}{w} P(z)$ and $P_{2}(z)=\frac{1}{b w} P(z)$.

In contrast to Ozawa's problem and complementing the three examples above, our primary focus is on exponential polynomial solutions of linear differential equations, in particular of second order equations

$$
f^{\prime \prime}+A(z) f^{\prime}+B(z) f=0
$$

where $A(z)$ and $B(z)$ are entire. An exponential polynomial is a function of the form

$$
f(z)=P_{1}(z) e^{Q_{1}(z)}+\cdots+P_{k}(z) e^{Q_{k}(z)},
$$


where $P_{j}, Q_{j}$ are polynomials for $1 \leq j \leq k$. Observe that a polynomial is a special case of an exponential polynomial. A transcendental exponential polynomial $f$ can be written in the normalized form

$$
f(z)=F_{0}(z)+F_{1}(z) e^{w_{1} z^{q}}+\cdots+F_{m}(z) e^{w_{m} z^{q}},
$$

where $q=\max \left\{\operatorname{deg}\left(Q_{j}\right)\right\} \geq 1$ is the order of $f$, the frequencies $w_{j}$ are non-zero and pairwise distinct, the multipliers $F_{j}$ are exponential polynomials of order $\leq q-1$ such that $F_{j}(z) \not \equiv 0$ for $1 \leq j \leq m$, and $m \leq k$ [8, 16].

Definition $1.4([18])$ Let $f$ be given in the normalized form (1.5). If the non-zero frequencies $w_{1}, \ldots, w_{m}$ of $f$ all lie on a fixed ray $\arg (w)=\theta$, then $f$ is called a simple exponential polynomial. If $g$ is another simple exponential polynomial of the same order $q$ as $f$ such that the non-zero frequencies of $g$ all lie on the opposite ray $\arg (w)=\theta+\pi$, then $f$ and $g$ are called dual exponential polynomials.

For example, the functions $f(z)=z^{2} e^{-i z}+z e^{z^{2}}+e^{2 z^{2}+(1-i) z}$ and $g(z)=2 e^{-z^{2}+(1+i) z}+$ $z^{2} e^{-4 z^{2}+i z}$ are dual exponential polynomials of order 2 .

In studying the differential equation (1.3) with entire coefficients $A(z)$ and $B(z)$, it is fundamental that each of its solutions $f$ is an entire function also. In this paper we study cases when $f$ can be an exponential polynomial assuming that $A(z)$ is an exponential polynomial and that $B(z)$ grows slowly compared to $A(z)$. Naturally, the set $\mathcal{E}$ of entire functions is a ring closed under differentiation, and the set $\operatorname{Exp}_{q}$ of exponential polynomials of order $\leq q \in \mathbb{N}$ together with constants in $\mathbb{C}$ and ordinary polynomials in $\mathbb{C}[z]=$ : $\operatorname{Exp}_{0}$ becomes a differential subring of $\mathcal{E}$. On the other hand, $\operatorname{Exp}_{q}$ is not closed under integration in general except for the set $\operatorname{Exp}_{1}$ of exponential polynomials of order $\leq 1$, which plays a role in our discussions.

To identify a primitive of each element in $\operatorname{Exp}_{1}$, it is convenient to use the formula

$$
\int z^{n} e^{w z} d z=\left(\frac{1}{w} z^{n}+\sum_{\nu=0}^{n-1} \frac{(-1)^{n-\nu} n !}{w^{n-\nu+1} \nu !} z^{\nu}\right) e^{w z}+\text { constant }
$$

for $n \in \mathbb{N} \cup\{0\}$ and $w \in \mathbb{C} \backslash\{0\}$. Of course, an analogous formula is not in general available for $z^{n} e^{w z^{q}}$ when $q \geq 2$. Indeed, recall the error function $\operatorname{erf}(z)$ defined also for complex argument $z$ by

$$
\operatorname{erf}(z)=\frac{2}{\sqrt{\pi}} \int_{0}^{z} e^{-\zeta^{2}} d \zeta
$$

It is the primitive of $\frac{2}{\sqrt{\pi}} e^{-z^{2}} \in \operatorname{Exp}_{2}$, but the function itself is not an exponential polynomial. For this reason one needs the special expression $\operatorname{erf}(z)$ for this function as in the real argument case. This is also the case when $q \geq 3$. The value distribution of the functions $\int_{0}^{z} e^{-\zeta^{q}} d \zeta, q \in \mathbb{N}$, as described in Nevanlinna's monograph [14, pp. 168-170], is quite different from that of exponential polynomials [8, 10, 16].

Along with $\operatorname{Exp}_{q-1}$, the set $\mathcal{S}_{q}(\theta)$ of simple exponential polynomials of order $q$ with respect to a fixed angle $\theta \in[0,2 \pi)$ forms a differential subring of $\operatorname{Exp}_{q}$. A unit element in $\mathcal{S}_{q}(\theta)$ is a single exponential term $e^{w z^{q}+p(z)}$ with $\arg (w)=\theta, p(z) \in \mathbb{C}[z]$ and $\operatorname{deg}(p) \leq$ $q-1$, whose multiplicative inverse belongs to the set $\mathcal{S}_{q}(\theta+\pi)$ as its dual exponential polynomial. It should be observed that if $f \in \mathcal{S}_{q}(\theta)$ and $g \in \mathcal{S}_{q}(\theta+\pi)$ are dual exponential polynomials, then $f g \in \operatorname{Exp}_{q-1}$ might not hold, but even so, the growth of $f g$ in terms of the characteristic function could be somewhat reduced from that of $f$ or $g$. 
Example 1.5 If $f(z)=e^{z}+e^{2 z}$ and $g(z)=e^{-4 z}$, then $T(r, f)=\frac{2}{\pi} r+O(\log r)$ and $T(r, g)=\frac{4}{\pi} r+O(\log r)$, while $T(r, f g)=\frac{3}{\pi} r+O(\log r)$, see [10]. Alternatively, the choice $g(z)=e^{-z}$ gives $T(r, g)=\frac{1}{\pi} r$ and $T(r, f g)=\frac{1}{\pi} r+O(1)$.

In our setting, the duality of two exponential polynomials is an interdependence among them in order to reduce the growth under multiplication, especially when combined with differentiation. For example, if $A$ and $f$ are dual exponential polynomials of order $q$, then $A$ and $f^{\prime}$ are also dual, and at times the growth of $A f^{\prime}$ is reduced to $\rho\left(A f^{\prime}\right)<q$.

The motivation for studying exponential polynomial solutions of (1.3) arises from the following previous result.

Theorem 1.6 ([18]) Suppose that $f$ is a transcendental exponential polynomial solution of (1.3), where $A(z)$ and $B(z)$ are exponential polynomials satisfying $\rho(B)<\rho(A)$. Then the following assertions hold.

(a) $f$ and $A(z)$ are dual exponential polynomials of order $q \in \mathbb{N}$, and $f$ has the normalized representation

$$
f(z)=c+F_{1}(z) e^{w_{1} z^{q}}+\cdots+F_{m}(z) e^{w_{m} z^{q}},
$$

where $m \in \mathbb{N}$ and $c \in \mathbb{C} \backslash\{0\}$.

(b) If $\rho\left(A f^{\prime}\right)<q$, then $q=1$ and

$$
A(z)=a e^{-w z}, \quad B(z)=-w^{2} \quad \text { and } \quad f(z)=c\left(1+\frac{w}{a} e^{w z}\right)
$$

where $w=w_{1}$ and $a \in \mathbb{C} \backslash\{0\}$.

If $a=c=w=1$, then (1.7) reduces to Frei's equation (1.1) and Frei's solution (1.2) in the case $m=1$. The following example illustrates that it is not always the case that the differential equation (1.3) possesses a non-trivial exponential polynomial solution when $A(z)$ and $B(z)$ are exponential polynomials satisfying $\rho(B)<\rho(A)$.

Example 1.7 For a fixed $n \in \mathbb{Z}$, let $A(z)=-\frac{5}{3}+n+\frac{2}{3} e^{-z}$ and $B(z)=-\frac{8}{3}+n$. Then (1.3) has a zero-free solution

$$
f(z)=\exp \left\{\frac{2}{3} e^{-z}+\left(\frac{8}{3}-n\right) z\right\} .
$$

Note that $f$ is an exponential of an exponential polynomial. Another solution of (1.3), linearly independent with $f$, is

$$
\begin{aligned}
g(z) & =f(z) \int^{z} \frac{e^{-\zeta}}{f(\zeta)} d \zeta \\
& =\exp \left\{\frac{2}{3} e^{-z}+\left(\frac{8}{3}-n\right) z\right\} \int^{z} \exp \left\{\frac{2}{3} e^{-\zeta}+\left(\frac{5}{3}-n\right) \zeta\right\} d \zeta
\end{aligned}
$$

where the integral represents an arbitrary primitive function. We may re-write this as

$$
g(z) \exp \left\{-\frac{2}{3} e^{-z}-\left(\frac{8}{3}-n\right) z\right\}=\int^{z} \exp \left\{\frac{2}{3} e^{-\zeta}+\left(\frac{5}{3}-n\right) \zeta\right\} d \zeta
$$


to see that $g$ solves a first order equation

$$
g^{\prime}(z)+\left\{-\frac{2}{3} e^{-z}+\left(\frac{8}{3}-n\right)\right\} g(z)=\exp \left\{\frac{4}{3} e^{-z}+\left(\frac{13}{3}-2 n\right) z\right\} .
$$

This shows that $g$ cannot be any exponential polynomial as a function of infinite order. Hence it is necessary in Theorem 1.6 to assume that (1.3) has a nontrivial exceptional polynomial solution $f$.

One may also observe that a small perturbation in the above coefficients $A(z)$ and $B(z)$ brings our desired case. In fact, by choosing $A(z)=-\frac{5}{3}-n+\frac{2}{3} e^{-z}$ and $B(z)=-\frac{2}{3}+2 n$ for any $n \in \mathbb{Z}$, the equation (1.3) permits the exponential polynomial solution

$$
f(z)=1+(1-3 n) e^{z}+(1-3 n)\left(1-\frac{3}{4} n\right) e^{2 z} .
$$

A difference between these two cases can also be observed in the logarithmic derivatives: If $f$ is the function in (1.8), then $\frac{f^{\prime}(z)}{f(z)}=-\frac{2}{3} e^{-z}+\frac{8}{3}-n$, while if $f$ is the function in (1.9), then $\frac{f^{\prime}(z)}{f(z)}$ is not an exponential polynomial but an irreducible rational function in $e^{z}$.

After discussing some properties of exponential polynomials in Section 2, we will show in Section 3 that the conclusions in Theorem 1.6(a) can be made stronger under weaker assumptions. Complementing the condition $\rho\left(A f^{\prime}\right)<q$ in Theorem 1.6(b), some new conditions implying the conclusion $q=1$ will be discovered. Examples on higher order duality as well as on the cases where a solution is dual to more than one coefficient will be discussed in Sections 4 and 5, respectively. Two open problems are formulated in the hope that these findings would give raise to further discussions in the future.

\section{Preliminaries on exponential polynomials}

We need to introduce several concepts some of which are new.

Definition 2.1 ([11, p. 214]) Let $f$ in (1.5) be a simple exponential polynomial. If there exists a constant $w \in \mathbb{C} \backslash\{0\}$ such that $w_{j} / w$ is a positive integer for every $j=1,2, \ldots, m$, then the (non-zero) frequencies of $f$ are said to be commensurable, and $w$ is called a common factor.

For example, $f(z)=e^{\pi z}+3 e^{2 \pi z}+z e^{3 \pi z}$ and $g(z)=e^{4 i z}+e^{6 i z}$ are simple exponential polynomials, both of their frequencies are commensurable, and examples for common factors are $\pi, \pi / 2$ for $f$ and $i, 2 i$ for $g$. In particular, a common factor is not unique.

Note that it is usual to say that non-zero real numbers $a$ and $b$ are commensurable if their ratio $a / b$ is a rational number. Equivalently, there exist a real number $c$ and integers $m$ and $n$ such that $a=m c$ and $b=n c$. In Definition 2.1 we are concerned with a simple exponential polynomial and a fixed $\theta \in[0,2 \pi)$, and thus all the non-zero frequencies are of the form $w_{j}=r_{j} e^{i \theta}$ for $r_{j}>0$, and the ratio of $w_{j}$ and $w_{i}$ is

$$
\frac{w_{j}}{w_{i}}=\frac{r_{j}}{r_{i}}=\frac{w_{j} / w}{w_{i} / w}
$$

This is a positive rational number for a common factor $w$. If we consider the dual exponential polynomial as well, all those frequencies are commensurable in the usual sense. 
If $f$ is a simple exponential polynomial of order one with constant multipliers, and if its frequencies are commensurable as in Frei's case (1.2), then by the fundamental theorem of algebra, $f$ can be written as

$$
f(z)=A \prod_{j=1}^{m}\left(e^{w z}-\alpha_{j}\right)
$$

where $A \neq 0, \alpha_{j}$ 's are complex constants and $m$ is a positive integer. In particular, all the zeros of $f$ lie on at most $m$ lines.

We note that if the non-zero frequencies of $f$ are commensurable, then they are clearly linearly dependent over rationals (see [13] for results in this direction), but not the other way around. For example, the points $w_{1}=1, w_{2}=\sqrt{2}, w_{3}=\sqrt{2}-1$ are linearly dependent over rationals but not commensurable.

Definition 2.2 Suppose that $f$ and $g$ are dual exponential polynomials with commensurable frequencies $\left\{w_{j}\right\}(j>0)$ and $\left\{\lambda_{i}\right\}(i>0)$, respectively, sharing the same common factor $w$ but with opposite signs. If the points $w_{j}+\lambda_{i}$ are on one ray including the origin for all $i, j>0$, then $f$ and $g$ are called strongly dual exponential polynomials.

For example, the functions $f(z)=1+z e^{z}+2 e^{3 z}$ and $g(z)=1-e^{-z}$ are strongly dual exponential polynomials, while $f(z)$ and $h(z)=g(z)+2 z^{2} e^{-2 z}$ are not. Note that if $\arg \left(w_{j}\right)=\theta$, then $\arg \left(\lambda_{j}\right)=\theta+\pi$ by duality, and moreover, if $w_{j}+\lambda_{i} \neq 0$, then precisely one of $\arg \left(w_{j}+\lambda_{i}\right)=\theta$ or $\arg \left(w_{j}+\lambda_{i}\right)=\theta+\pi$ holds for all $i, j>0$. Alternatively, strong duality of $f$ and $g$ of order $q$ can be expressed as follows: There exists a non-zero constant $w$ such that

$$
f(z)=\sum_{j=0}^{m} F_{j}(z)\left(e^{w z^{q}}\right)^{j} \quad \text { and } \quad g(z)=\sum_{i=0}^{m} G_{i}(z)\left(e^{-w z^{q}}\right)^{i}
$$

where $F_{j}, G_{i}$ are exponential polynomials of order $\leq q-1$. Hence $f$ is a polynomial in $e^{w z^{q}}$ and $g$ is a polynomial in $e^{-w z^{q}}$, with smaller exponential polynomials as multipliers. Using the notation above, $f \in \operatorname{Exp}_{q-1}\left[e^{w z^{q}}\right]$ and $g \in \operatorname{Exp}_{q-1}\left[e^{-w z^{q}}\right]$. Differing from the situation in (1.5), some of the multipliers $F_{j}, G_{i}(i, j>0)$ in (2.1) must suitably vanish identically so that only one of $j-i \geq 0$ or $j-i \leq 0$ always holds for all non-vanishing multipliers $F_{j}, G_{i}(i, j>0)$. This is a consequence of Definition 2.2 .

We may think that being strong in our duality means that the product of $f(z)-F_{0}(z)$ and $g(z)-G_{0}(z)$ becomes again a commensurable exponential polynomial with either $w$ or $-w$ as a common factor. In the case when both $F_{0}(z)$ and $G_{0}(z)$ are constant, each product of the derivatives $f^{(k)}(z)$ and $g^{(\ell)}(z), k, \ell \in \mathbb{N}$, is a commensurable exponential polynomial with the same common factor as the product of $f(z)-F_{0}(z)$ and $g(z)-G_{0}(z)$.

Definition 2.3 ([18]) Denote the set of complex conjugate frequencies of the function $f$ in (1.5) by $W_{f}=\left\{\bar{w}_{0}, \bar{w}_{1}, \ldots, \bar{w}_{m}\right\}$, where $\bar{w}_{0}=0$ is related to the multiplier $F_{0}(z) \not \equiv 0$, and $W_{f}=\left\{\bar{w}_{1}, \ldots, \bar{w}_{m}\right\}$ when $F_{0}(z) \equiv 0$. Denote the convex hull of the set $W_{f}$ by $\operatorname{co}\left(W_{f}\right)$, and let $C\left(\operatorname{co}\left(W_{f}\right)\right)$ denote the circumference of $\operatorname{co}\left(W_{f}\right)$.

The set $\operatorname{co}\left(W_{f}\right)$ is defined as the intersection of all closed convex sets containing $W_{f}$, and as such it is either a convex polygon or a line segment. The latter occurs when $f$ is simple, and, in particular, when $w_{1}, \ldots, w_{m}$ are commensurable. The vertices of co $\left(W_{f}\right)$ are formed by some (possibly all) of the points $\bar{w}_{0}, \bar{w}_{1}, \ldots, \bar{w}_{m}$. The circumference $C\left(\operatorname{co}\left(W_{f}\right)\right)$ of co $\left(W_{f}\right)$ plays an important role in describing the value distribution of $f$, see [8, 10, 16]. 
Let $h$ be a quotient of two transcendental exponential polynomials, say

$$
h(z)=f(z) / g(z)
$$

where $f$ is of the form (1.5) and $g$ is an exponential polynomial of the normalized form

$$
g(z)=G_{0}(z)+G_{1}(z) e^{w_{1} z^{q}}+\cdots+G_{m}(z) e^{w_{m} z^{q}} .
$$

In these representations of $f$ and $g$ for the quotient $h$, we allow that some of the multipliers $F_{j}$ or $G_{j}$ may vanish identically, but we suppose that the matching multipliers $F_{j}$ and $G_{j}$ do not both vanish identically for any $j$.

For the quotient $h=f / g$, define the set $W_{h}=\left\{\bar{w}_{0}, \bar{w}_{1}, \ldots, \bar{w}_{m}\right\}$. The proximity function of $h$ is

$$
m(r, h)=\left(C\left(\operatorname{co}\left(W_{h}\right)\right)-C\left(\operatorname{co}\left(W_{g}\right)\right)\right) \frac{r^{q}}{2 \pi}+o\left(r^{q}\right),
$$

see [17, Satz 1]. In particular, if $g \equiv 1$, then $W_{g}=\{0\}$ and $C\left(\operatorname{co}\left(W_{g}\right)\right)=0$. This yields [16, Satz 1] as a special case, namely

$$
T(r, f)=m(r, f)=C\left(\operatorname{co}\left(W_{f}^{0}\right)\right) \frac{r^{q}}{2 \pi}+o\left(r^{q}\right),
$$

where $W_{f}^{0}=W_{f} \cup\{0\}$. The estimates (2.2) and (2.3) are consistent with the estimate

$$
m\left(r, \frac{f^{\prime}}{f}\right)=o(T(r, f)),
$$

known as the lemma on the logarithmic derivative, since $W_{f^{\prime} / f}=W_{f}$ holds for any given exponential polynomial $f$ of the form (1.5). We also point out that $W_{f / f^{\prime}}=W_{f^{\prime}}$. This fact will be used in proving our main results in Section 3 .

\section{The main results}

Motivated by Example 1.3, we improve Theorem[1.6(a) under weaker assumptions on $B(z)$.

Theorem 3.1 Suppose that $f$ and $A(z)$ in (1.3) are transcendental exponential polynomials, and that $B(z)$ is an entire function satisfying $T(r, B)=o(T(r, A))$. Then the following assertions hold.

(a) $f$ and $A(z)$ are dual exponential polynomials of order $q \in \mathbb{N}$, $f$ has the normalized representation (1.6), and $B(z)$ is an exponential polynomial of order $\rho(B) \leq q-1$.

(b) The frequencies of $f$ are commensurable if and only if the frequencies of $A(z)$ are commensurable. In both cases, $f$ and $A(z)$ are strongly dual exponential polynomials.

Proof. (a) Suppose that $0 \leq \rho(f) \leq \rho(A)-1$. The case $\rho(f)=0$ is not possible because $f$ is transcendental. Hence $\rho(f) \geq 1$. But now

$$
|A| \leq\left|f^{\prime \prime} / f^{\prime}\right|+|B|\left|f / f^{\prime}\right|
$$

and the assumption $T(r, B)=o(T(r, A))$ imply

$$
T(r, A)=m(r, A) \leq m(r, B)+O\left(r^{\rho(A)-1}\right)=o(T(r, A))
$$

which is a contradiction. Here we have used (2.2) for $h=f / f^{\prime}$ and $g=f^{\prime}$, as well as (2.3) for $A$ in place of $f$. The following two cases are also impossible by the proof of [9, Theorem 3.6]: 
(1) $\rho(f)=\rho(A)$ and either $F_{0}(z) \equiv 0$ or $F_{0}^{\prime}(z) \not \equiv 0$.

(2) $\rho(f) \geq \rho(A)+1$.

Thus $\rho(f)=\rho(A)=q \geq 1$ and $f$ has the representation (1.6).

We proceed to prove that $f$ and $A(z)$ are dual exponential polynomials. Using (1.3), we find that

$$
m\left(r, \frac{A f^{\prime}}{f}\right)=O(\log r)+m(r, B)=o(T(r, A))=o\left(r^{q}\right)
$$

The formula (7.3) in [18] should be replaced by this. Thus the formula (7.7) in [18] holds, and the reasoning in [18] shows that $f$ and $A(z)$ are dual exponential polynomials.

To complete the proof of (a), it suffices to prove that $B(z)$ is an exponential polynomial of order $\rho(B) \leq q-1$. Since the frequencies $w_{j}$ of $f$ are all on one ray, we may appeal to a rotation, and suppose that $w_{1}, \ldots, w_{m} \in \mathbb{R}_{+}$. By renaming the frequencies $w_{j}$, if necessary, we may further suppose that $0<w_{1}<\cdots<w_{m}$. Thus the dual coefficient must be of the form

$$
A(z)=A_{0}(z)+\sum_{j=1}^{k} A_{j}(z) e^{-\lambda_{j} z^{q}}
$$

where $A_{j}(z) \not \equiv 0$ for all $j \in\{1, \ldots, k\}$ and $\lambda_{1}, \ldots, \lambda_{k} \in \mathbb{R}_{+}$. Renaming the frequencies $\lambda_{j}$, if necessary, we may suppose that $0<\lambda_{1}<\cdots<\lambda_{k}$. Write

$$
f^{\prime}(z)=\sum_{j=1}^{m} G_{j}(z) e^{w_{j} z^{q}} \quad \text { and } \quad f^{\prime \prime}(z)=\sum_{j=1}^{m} H_{j}(z) e^{w_{j} z^{q}}
$$

where $G_{j}(z)=F_{j}^{\prime}(z)+q w_{j} z^{q-1} F_{j}(z) \not \equiv 0$ and $H_{j}(z)=G_{j}^{\prime}(z)+q w_{j} z^{q-1} G_{j}(z) \not \equiv 0$. Next, write $-A f^{\prime}=B f+f^{\prime \prime}$ in the form

$$
-\left(\sum_{j=1}^{k} A_{j} e^{-\lambda_{j} z^{q}}\right)\left(\sum_{j=1}^{m} G_{j} e^{w_{j} z^{q}}\right)=c B+\sum_{j=1}^{m}\left(A_{0} G_{j}+B F_{j}+H_{j}\right) e^{w_{j} z^{q}} .
$$

From (3.2) we find that $B$ is an exponential polynomial of order $\rho(B) \leq q$. In fact, from (2.3) and the assumption $T(r, B)=o(T(r, A))$, it follows that $\rho(B) \leq q-1$.

(b) We begin with some preparations. From [16] and [17], we have

$$
m\left(r, \sum_{j=1}^{k} A_{j} e^{-\lambda_{j} z^{q}}\right)=T\left(r, \sum_{j=1}^{k} A_{j} e^{-\lambda_{j} z^{q}}\right)=\frac{\lambda_{k}}{\pi} r^{q}+o\left(r^{q}\right),
$$

and

$$
\begin{gathered}
m\left(r,\left\{c B+\sum_{j=1}^{m}\left(A_{0} G_{j}+B F_{j}+H_{j}\right) e^{w_{j} z^{q}}\right\} / \sum_{j=1}^{m} G_{j} e^{w_{j} z^{q}}\right) \\
=\frac{2 w_{m}-2\left(w_{m}-w_{1}\right)}{2 \pi} r^{q}+o\left(r^{q}\right)=\frac{w_{1}}{\pi} r^{q}+o\left(r^{q}\right) .
\end{gathered}
$$

Therefore, we deduce that

$$
0<\lambda_{1}<\cdots<\lambda_{k}=w_{1}<\cdots<w_{m}
$$


Thus from (3.2), it follows that

$$
-A_{k} G_{1}=c B .
$$

If $A_{0} G_{m}+B F_{m}+H_{m} \not \equiv 0$, then from [10, Theorem 2.2] and (3.3), we get

$$
\begin{aligned}
N(r, 0, L) & =\frac{2\left(w_{m}-w_{1}\right)+2\left(\lambda_{k}-\lambda_{1}\right)}{2 \pi} r^{q}+O\left(r^{q-1}+\log r\right) \\
& =\frac{w_{m}-\lambda_{1}}{\pi} r^{q}+O\left(r^{q-1}+\log r\right), \\
N(r, 0, R) & =\frac{w_{m}}{\pi} r^{q}+O\left(r^{q-1}+\log r\right),
\end{aligned}
$$

where $N(r, 0, L)$ and $N(r, 0, R)$ are the counting functions of zeros of the exponential polynomials on the left-hand side and on the right-hand side of (3.2), respectively. This implies $w_{m}=w_{m}-\lambda_{1}$, which is impossible. Thus we have

$$
A_{0} G_{m}+B F_{m}+H_{m} \equiv 0 .
$$

Now (3.2) reduces to

$$
-\left(\sum_{j=1}^{k} A_{j} e^{-\lambda_{j} z^{q}}\right)\left(\sum_{j=1}^{m} G_{j} e^{w_{j} z^{q}}\right)=c B+\sum_{j=1}^{m-1}\left(A_{0} G_{j}+B F_{j}+H_{j}\right) e^{w_{j} z^{q}} .
$$

From the Borel-Nevanlinna theorem, and from $A_{i} G_{j} \not \equiv 0$ for $j \in\{1,2, \ldots, m\}$ and $i \in$ $\{1,2, \ldots, k\}$, it follows that there are only two possibilities:

(I) For some pairs $(j, i)$, where $j \in\{1,2, \ldots, m\}$ and $i \in\{1,2, \ldots, k\}$, there exists $\ell \in\{0,1, \ldots, m-1\}$ such that

$$
w_{j}-\lambda_{i}=w_{\ell}
$$

(II) For some pairs $(j, i)$, where $j \in\{1,2, \ldots, m\}$ and $i \in\{1,2, \ldots, k\}$, there exist $s \in\{1,2, \ldots, m\} \backslash\{j\}$ and $t \in\{1,2, \ldots, k\} \backslash\{i\}$ such that

$$
w_{j}-\lambda_{i}=w_{s}-\lambda_{t}
$$

After these preparations we proceed to prove that the frequencies of $f$ are commensurable if and only if the frequencies of $A(z)$ are commensurable. By appealing to (3.3) and to a change of variable as in Example 1.3. we may suppose that $w_{1}=\lambda_{k} \in \mathbb{N}$. Thus we prove that $w_{j} \in \mathbb{N}$ for $j \in\{1, \ldots, m\}$ if and only if $\lambda_{i} \in \mathbb{N}$ for $i \in\{1, \ldots, k\}$.

(i) Suppose that $w_{j} \in \mathbb{N}$ for $j \in\{1, \ldots, m\}$. From (3.3), we see that $w_{m}-\lambda_{1}=$ $\max _{j, i}\left\{w_{j}-\lambda_{i}\right\}$ and $w_{m}-\lambda_{1}>w_{j}-\lambda_{i}$ for any $j \neq m$ and $i \neq 1$. Hence, from (3.7) and (3.8), there exists $p<m$ such that $w_{m}-\lambda_{1}=w_{p}$, which implies that $\lambda_{1} \in \mathbb{N}$. In addition, from (3.3), we have $w_{m}-\lambda_{2}>w_{j}-\lambda_{i}$ for any $j \neq m$ and $i>2$. Thus, from (3.7) and (3.8), there are only two possibilities: (1) There exists $p<m$ such that $w_{m}-\lambda_{2}=w_{p}-\lambda_{1}$. (2) There exists $p<m$ such that $w_{m}-\lambda_{2}=w_{p}$. In both cases, it follows that $\lambda_{2} \in \mathbb{N}$. Repeating this argument for $k$ times gives us $\lambda_{i} \in \mathbb{N}$ for $i \in\{1, \ldots, k\}$. 
(ii) Suppose that $\lambda_{i} \in \mathbb{N}$ for $i \in\{1, \ldots, k\}$. From (3.3), we have $\lambda_{k}=w_{1}$, and consequently $w_{1} \in \mathbb{N}$. Moreover, from (3.3), we have $w_{2}-\lambda_{k}<w_{j}-\lambda_{i}$ for any $j>1$ and $i \neq k$. Thus, from (3.7) and (3.8), there are only two possibilities: There exists $p<k$ such that either $w_{2}-\lambda_{k}=w_{1}-\lambda_{p}$ or $w_{2}-\lambda_{k}=w_{1}$. In both cases, we have $w_{2} \in \mathbb{N}$. Repeating this argument for $m$ times gives us $w_{j} \in \mathbb{N}$ for $i \in\{1, \ldots, m\}$.

If the frequencies are commensurable for one of $f, A(z)$, then they are commensurable for both of $f, A(z)$ by the reasoning above. The remaining fact that $f$ and $A(z)$ are strongly dual exponential polynomials now follows by (3.3. $)$.

The assumption $\rho\left(A f^{\prime}\right)<\rho(f)$ in Theorem 1.6(b) seems to be the only known sufficient condition for the conclusion $q=1$. However, in the case of Frei's result (1.1), we have

$$
A(z) f^{\prime}(z)=e^{-z} \sum_{j=1}^{m} j C_{j} e^{j z}=\sum_{j=0}^{m-1}(j+1) C_{j+1} e^{j z}
$$

and so $\rho\left(A f^{\prime}\right)=\rho(f)=1$. This shows that $q=1$ may happen even if $\rho\left(A f^{\prime}\right)=\rho(f)$. Theorem 3.2 below shows that $f$ having only one large exponential term is also a sufficient condition for $q=1$. In contrast, if $A(z)$ has only one large exponential term, then $f$ can have multiple large exponential terms as in (1.1).

Theorem 3.2 Suppose that $f(z)=F_{0}(z)+F_{1}(z) e^{w z^{q}}$ is a solution of (1.3), where $A(z)$ is an exponential polynomial and $B(z)$ is an entire function satisfying $T(r, B)=o(T(r, A))$. Then $q=1$, and there are constants $c, b \in \mathbb{C} \backslash\{0\}$ and a non-trivial polynomial $P(z)$ such that

$$
f(z)=c+b e^{w z}, A(z)=\frac{b}{c} P(z)-w+P(z) e^{-w z} \text { and } B(z)=-\frac{w b}{c} P(z) .
$$

Proof. We proceed similarly as in the proof of Theorem 3.1 until (3.6), which now reduces to the form

$$
-\left(\sum_{j=1}^{k} A_{j} e^{-\lambda_{j} z^{q}}\right) G_{1} e^{w z^{q}}=c B,
$$

where $F_{0}(z) \equiv c \in \mathbb{C} \backslash\{0\}$. Hence $k=1$, and consequently $A(z)$ reduces to the form

$$
A(z)=A_{0}(z)+A_{1}(z) e^{-w z^{q}} .
$$

From (3.9) and (3.5), with $k=1=m$, we find that

$$
-A_{1} G_{1}=c B \text { and } \quad-A_{0} G_{1}=B F_{1}+H_{1} .
$$

In other words,

$$
c^{-1} A_{1} G_{1} F_{1}=A_{0} G_{1}+H_{1}=A_{0} G_{1}+G_{1}^{\prime}+q w z^{q-1} G_{1} .
$$

Dividing both sides of (3.10) by $G_{1}$, we observe that at every zero of $G_{1}$ the right-hand side has a pole but the left-hand side does not. Thus $G_{1}$ has no zeros, and so we may write it in the form $G_{1}=e^{g}$, where $g(z)=a_{q-1} z^{q-1}+\cdots+a_{0}$ is a polynomial of degree $\leq q-1$. Since

$$
G_{1}=F_{1}^{\prime}+q w z^{q-1} F_{1}=e^{g}
$$


we obtain $\left(F_{1}(z) e^{w z^{q}}\right)^{\prime}=e^{w z^{q}+g(z)}$, and consequently

$$
F_{1}(z) e^{w z^{q}}=\int^{z} e^{w \zeta^{q}+a_{q-1} \zeta^{q-1}+\cdots+a_{0}} d \zeta
$$

Here the right-hand side is an exponential polynomial, which happens only if $q=1$.

Since $q=1$, we see from (3.11) that $F_{1}(z)$ reduces to a non-zero constant, say $F_{1}(z) \equiv b$. Thus $f(z)=c+b e^{w z}$, and we have $G_{1}(z) \equiv w b$ and $H_{1}(z) \equiv w^{2} b$. A substitution to (3.10) followed by a simplification gives

$$
\frac{b}{c} A_{1}=A_{0}+w
$$

There is no restriction for $A_{1}$ other than the fact that $A$ is an exponential polynomial. Thus we may suppose that $A_{1}$ is any non-trivial polynomial, say $A_{1}=P$. This gives us $A_{0}=\frac{b}{c} P-w$, and finally $B=-\frac{w b}{c} P$.

Example 1.1 shows that the coefficient $B(z)$ in (1.3) can be a polynomial. Next, we prove that this is equivalent to $A_{0}(z)$ in (3.1) being a polynomial, and reveal another sufficient condition for the conclusion $q=1$.

Proposition 3.3 Under the assumptions of Theorem 3.1, the term $A_{0}(z)$ of $A(z)$ in (3.1) is a polynomial if and only if $B(z)$ is a polynomial. Moreover, if the multipliers of $f$ and of $A(z)$ are constants, then $q=1$ and $B(z)$ is a constant function.

Proof. From the proof of Theorem 3.1 we find that (3.5) holds, that is,

$$
A_{0} G_{m}+B F_{m}+H_{m}=0
$$

where

$$
\begin{aligned}
& G_{m}=F_{m}^{\prime}+w_{m} q z^{q-1} F_{m}, \\
& H_{m}=F_{m}^{\prime \prime}+2 w_{m} q z^{q-1} F_{m}^{\prime}+\left(w_{m} q(q-1) z^{q-2}+w_{m}^{2} q^{2} z^{2 q-2}\right) F_{m} .
\end{aligned}
$$

Thus $F_{m}$ solves the second order differential equation

$$
F_{m}^{\prime \prime}+P(z) F_{m}^{\prime}+Q(z) F_{m}=0
$$

where

$$
\begin{aligned}
& P(z)=2 w_{m} q z^{q-1}+A_{0}, \\
& Q(z)=w_{m} q z^{q-1} A_{0}+w_{m} q(q-1) z^{q-2}+w_{m}^{2} q^{2} z^{2(q-1)}+B .
\end{aligned}
$$

Suppose first that $A_{0}(z)$ is a polynomial. If $B(z)$ is transcendental, then it follows from (3.13) and [5, Corollary 1] that $\rho\left(F_{m}\right)=\infty$, which is a contradiction. Hence $B(z)$ must be a polynomial. Conversely, suppose that $B(z)$ is a polynomial. Suppose on the contrary to the assertion that $A_{0}(z)$ is a transcendental exponential polynomial. Then there exists an open sector $S$ such that $A_{0}(z)$ blows up exponentially in $S$. Using [6, Corollary 1] and $\rho\left(F_{m}\right) \leq q-1$ in (3.13), we obtain on almost every ray in $S$ that

$$
\left|w_{m} q z^{q-1}\right|\left|A_{0}(z)\right| \leq O\left(|z|^{\max \{2 q, \operatorname{deg}(B)\}}\right)+O\left(|z|^{q-2+\varepsilon}\left|A_{0}(z)\right|\right) .
$$

However, this is obviously a contradiction, and hence $A_{0}(z)$ is a polynomial. 
Finally, suppose that the multipliers of $f$ and of $A(z)$ are constants. From (3.4), we find that $B(z)=C z^{q-1}$ for some constant $C \in \mathbb{C} \backslash\{0\}$. Since $F_{m}(z)$ is a non-zero constant function, it follows that the coefficient $Q(z)$ in (3.13) vanishes identically. But this is not possible because $A_{0}(z)$ is a constant function, unless $q=1$.

Remark. (a) The equation (3.13) implies that every possible zero of $F_{m}$ is simple.

(b) Assuming that $A_{0}(z)$ is a polynomial, we give an alternative proof for the fact that $B(z)$ is a polynomial. We already know from Theorem 3.1 that $B(z)$ is of order $\leq q-1$. Since the non-zero frequencies of $A(z)$ are all on one ray by duality, it follows that the plane divides into $2 q$ sectors of opening $\pi / q$ such that in every other sector $A(z)$ either blows up exponentially or is asymptotic to the polynomial $A_{0}(z)$. In the latter case, if $A_{0}(z) \equiv 0$, then $A(z)$ decays to zero exponentially. Thus, from [5, Theorem 7], we deduce that $B(z)$ is a polynomial. Note, in particular, that the constant $\mu$ in [5, Theorem 7] satisfies $\mu=\pi / q$.

Open problem 1. Under the assumptions of Theorem 3.1, is it always true that $q=1$ and $B(z)$ is a polynomial?

This problem is fragile in the sense that the desired conclusion is not valid if a minor modification in the assumptions of Theorem 3.1 is performed. For example, the differential equation

$$
f^{\prime \prime}-\left(q w z^{q-1}+z^{-1} e^{-w z^{q}}\right) f^{\prime}-q(q-1) w z^{q-2} f=0
$$

possesses an exponential polynomial solution $f(z)=e^{w z^{q}}-1 /(q-1)$ for any $q \geq 2$. Moreover, the function $f(z)=e^{z^{2}}+1$ satisfies the differential equations

$$
\begin{array}{r}
f^{\prime \prime}+\left(\frac{e^{-z^{2}}-1}{2 z}-2 z\right) f^{\prime}-f=0, \\
f^{\prime \prime}-\frac{e^{-z^{2}}(z-1)+4 z^{2}+z+1}{2 z} f^{\prime}+(z-1) f=0 .
\end{array}
$$

The transcendental coefficients in (3.14) are entire exponential polynomials with rational multipliers because $z=0$ is a removable singularity for both.

\section{Duality for higher order functions}

Next we construct examples of differential equations of order $n \geq 2$ having an exponential polynomial solution $f$ of order $\rho(f)=n-1$ which is dual with one of the coefficients.

Example 4.1 If $H$ is an arbitrary entire function, then $f(z)=e^{z^{2}}+1$ solves

$$
\begin{array}{r}
f^{\prime \prime \prime}+\left(1+e^{-z^{2}}\right) H f^{\prime \prime}-\left(6+4 z^{2}\right) f^{\prime}-\left(2+4 z^{2}\right) H f=0, \\
f^{\prime \prime \prime}-2 z f^{\prime \prime}+\left(H-4+H e^{-z^{2}}\right) f^{\prime}-2 z H f=0 .
\end{array}
$$

A particularly interesting case is when $H$ is either a polynomial or an exponential polynomial of order one. Thus either of the two possible coefficients can be dual with $f$. Examples of second order dual solutions for third order equations can be found in [18] but for polynomial coefficients only. 
We can use the relation $z f^{\prime \prime}(z)=\left(2 z^{2}+1\right) f^{\prime}$ to see that, in addition to (4.1), the function $f(z)=e^{z^{2}}+1$ satisfies the equations

$$
\begin{aligned}
f^{\prime \prime \prime}(z)-2 z f^{\prime}(z)-4 f^{\prime}(z) & =0, \\
\left(1+e^{-z^{2}}\right) f^{\prime}(z)-2 z f(z) & =0, \\
\left(1+e^{-z^{2}}\right) f^{\prime \prime}(z)-\left(2+4 z^{2}\right) f(z) & =0 .
\end{aligned}
$$

Example 4.2 If $H$ is an arbitrary entire function, then $f(z)=e^{z^{3}}+1$ solves

$$
\begin{array}{r}
f^{(4)}+\left(1+e^{-z^{3}}\right) H f^{\prime \prime \prime}-9 z^{4} f^{\prime \prime}-30\left(2+3 z^{3}\right) f^{\prime}-\left(6+54 z^{3}+27 z^{6}\right) H f=0, \\
f^{(4)}-3 z^{2} f^{\prime \prime \prime}+\left(H-18 z+H e^{-z^{3}}\right) f^{\prime \prime}-18 f^{\prime}-H\left(6 z+9 z^{4}\right) f=0, \\
f^{(4)}-3 z^{2} f^{\prime \prime \prime}-27 z f^{\prime \prime}+\left(H+27 z^{3}+H e^{-z^{3}}\right) f^{\prime}-3 z^{2} H f=0 .
\end{array}
$$

A particularly interesting case is when $H$ is an exponential polynomial of order at most two. Thus all three of the possible coefficients can be dual with $f$. Previous examples of third order dual solutions do not seem to be known.

As in the previous example, we can use the relations $z f^{\prime \prime}(z)=\left(3 z^{3}+2\right) f^{\prime}(z)$ and $z f^{\prime \prime \prime}(z)=\left(3 z^{3}+1\right) f^{\prime \prime}(z)+9 z^{2} f^{\prime}(z)$ to see that $f(z)=e^{z^{3}}+1$ satisfies the equations

$$
\begin{aligned}
f^{(4)}(z)-3 z^{2} f^{\prime \prime \prime}(z)-18 z f^{\prime \prime}(z)-18 f^{\prime}(z) & =0, \\
\left(1+e^{-z^{3}}\right) f^{\prime}(z)-3 z^{2} f(z) & =0, \\
\left(1+e^{-z^{3}}\right) f^{\prime \prime}(z)-\left(6 z+9 z^{4}\right) f(z) & =0, \\
\left(1+e^{-z^{3}}\right) f^{\prime \prime \prime}(z)-\left(6+54 z^{3}+27 z^{6}\right) f(z) & =0 .
\end{aligned}
$$

In light of Open problem 1 and the examples just discussed, it is natural to pose our second open problem.

Open problem 2. If a solution and the dominant coefficient are dual exponential polynomials of order $q$, then is the differential equation in question of order at least $q+1$ ?

For the fragility of this problem, recall the equations (3.14) satisfied by $f(z)=e^{z^{2}}+1$. Moreover, the function $f(z)=e^{z^{3}}+1$ satisfies the third order equation

$$
f^{\prime \prime \prime}+\left(\frac{e^{-z^{3}}-1}{2 z}\right) f^{\prime \prime}-3 z\left(3 z^{3}+5\right) f^{\prime}-\frac{3}{2}\left(3 z^{3}+2\right) f=0
$$

with entire coefficients.

As the first initial step to knowing more about Open problem 2, we make a summary of the fundamental ideas in constructing Examples 4.1 and 4.2 .

Lemma 4.3 The function $f(z)=e^{z^{q}}+1, q \in \mathbb{N}$, possesses the following two properties:

(i) $\left(1+e^{-z^{q}}\right) f^{(j+1)}(z)=\sum_{k=0}^{j} P_{j, k}(z) f^{(k)}(z), \quad j \in \mathbb{N} \cup\{0\}$,

(ii) $f^{(q+1)}(z)=\sum_{\ell=1}^{q} Q_{\ell}(z) f^{(\ell)}(z)$, 
where the $P_{j, k}(z)$ and $Q_{\ell}(z)$ are non-zero polynomials satisfying

(a) $\left\{\begin{array}{l}P_{j+1, j+1}(z)=P_{j, j}(z)-q z^{q-1}, \quad P_{0,0}(z)=q z^{q-1}, \\ P_{j+1, k}(z)=P_{j, k}^{\prime}(z)+q z^{q-1} P_{j, k}(z)+P_{j, k-1}(z), \quad P_{j,-1}(z) \equiv 0, \quad 1 \leq k \leq j, \\ P_{j+1,0}(z)=P_{j, 0}^{\prime}(z)+q z^{q-1} P_{j, 0}(z),\end{array}\right.$

(b) $Q_{\ell}(z)=-\left(\begin{array}{c}q \\ \ell-1\end{array}\right)\left(e^{-z^{q}}\right)^{(q-\ell+1)} e^{z^{q}}$.

Proof. First, let us prove (i) by induction on $j$. Of course, by taking their logarithmic derivatives, we have $\left(1+e^{-z^{q}}\right) f^{\prime}(z)=q z^{q-1} f(z)$ immediately, that is, the case when $j=0$ follows with $P_{0,0}(z)=q z^{q-1}$. Assume (i) is true for each $j=0,1, \ldots, n$. Then

$$
\begin{aligned}
\left(1+e^{-z^{q}}\right) f^{(n+2)}(z)= & q z^{q-1} e^{-z^{q}} f^{(n+1)}+\sum_{k=0}^{n}\left\{P_{n, k}^{\prime}(z) f^{(k)}(z)+P_{n, k}(z) f^{(k+1)}(z)\right\} \\
= & q z^{q-1}\left(1+e^{-z^{q}}\right) f^{(n+1)}(z)+\left\{P_{n, n}(z)-q z^{q-1}\right\} f^{(n+1)}(z)+ \\
& +\sum_{k=1}^{n}\left\{P_{n, k}^{\prime}(z) f^{(k)}(z)+P_{n, k-1}(z)\right\} f^{(k)}(z)+P_{n, 0}^{\prime}(z) f(z) \\
= & q z^{q-1} \sum_{k=0}^{n} P_{n, k}(z) f^{(k)}(z)+\left\{P_{n, n}(z)-q z^{q-1}\right\} f^{(n+1)}(z)+ \\
& +\sum_{k=1}^{n}\left\{P_{n, k}^{\prime}(z) f^{(k)}(z)+P_{n, k-1}(z)\right\} f^{(k)}(z)+P_{n, 0}^{\prime}(z) f(z) \\
= & \left\{P_{n, n}(z)-q z^{q-1}\right\} f^{(n+1)}(z)+ \\
& +\sum_{k=1}^{n}\left\{P_{n, k}^{\prime}(z) f^{(k)}(z)+q z^{q-1} P_{n, k}(z)+P_{n, k-1}(z)\right\} f^{(k)}(z)+ \\
& +\left\{P_{n, 0}^{\prime}(z)+q z^{q-1} P_{n, 0}(z)\right\} f(z),
\end{aligned}
$$

which is the one to be proved.

Second, let us calculate the $q$-th order derivative of the product $f^{\prime}(z) e^{-z^{q}}=q z^{q-1}$. The Leibniz rule gives

$$
\sum_{\ell=0}^{q}\left(\begin{array}{l}
q \\
\ell
\end{array}\right) f^{(\ell+1)}(z)\left(e^{-z^{q}}\right)^{(q-\ell)} \equiv 0 .
$$

Denoting $Q_{\ell+1}(z)=-\left(\begin{array}{c}q \\ \ell\end{array}\right)\left(e^{-z^{q}}\right)^{(q-\ell)} e^{z^{q}}$ for $0 \leq \ell \leq q-1$, we have

$$
f^{(q+1)}(z)=\sum_{\ell=0}^{q-1} Q_{\ell+1}(z) f^{(\ell+1)}(z)=\sum_{\ell=1}^{q} Q_{\ell}(z) f^{(\ell)}(z)
$$

as desired.

Example 4.4 We may apply the two identities in Lemma 4.3 to construct differential equations of arbitrary order. Given any entire function $H$, we have the identity

$$
f^{(q+1)}(z)-\sum_{\ell=1}^{q} Q_{\ell}(z) f^{(\ell)}(z)=H(z)\left(\left(1+e^{-z^{q}}\right) f^{(j)}(z)-\sum_{k=0}^{j-1} P_{j-1, k}(z) f^{(k)}(z)\right),
$$


that is, $f(z)=e^{z^{q}}+1$ solves

$$
\begin{aligned}
f^{(q+1)}(z) & -\sum_{\ell=j+1}^{q} Q_{\ell}(z) f^{(\ell)}(z)-\left(\left(1+e^{-z^{q}}\right) H(z)+Q_{j}(z)\right) f^{(j)}(z) \\
& +\sum_{\ell=1}^{j-1}\left(P_{j-1, \ell}(z) H(z)-Q_{\ell}(z)\right) f^{(\ell)}(z)+P_{j-1,0}(z) H(z) f(z)=0
\end{aligned}
$$

where $1 \leq j \leq q$, the sum $\sum_{\ell=1}^{j-1}$ is empty if $j=1$ and the sum $\sum_{\ell=j+1}^{q}$ is empty if $j=q$.

\section{$5 \quad$ Multiple duality}

The possibility that a solution $f$ would be dual to more than one coefficient has not been studied rigorously. In this case there would be at least two equally strong dominant coefficients, or, in the case of (1.3), both coefficients $A(z), B(z)$ would be equally strong. For example, $f(z)=e^{-z}$ solves

$$
f^{\prime \prime}+e^{z} f^{\prime}+\left(e^{z}-1\right) f=0
$$

and is dual to both coefficients. Obviously the coefficients are not dual to each other. More examples can be produced from Example 1.1. Note that $f(z)=e^{z}$ solves (1.3) if $A(z)=-B(z)-1$. Hence $f$ is not necessarily dual with either of $A(z), B(z)$.

If $H$ is any entire function, then $f(z)=e^{z^{q}}$ solves

$$
f^{\prime \prime}+\left(H(z)-q z^{q-1}\right) f^{\prime}-\left(q(q-1) z^{q-2}+q z^{q-1} H(z)\right) f=0 .
$$

This example is from [5]. Note that $f(z)=e^{z^{q}}$ satisfies both $f^{\prime}(z)-q z^{q-1} f(z)=0$ and $f^{\prime \prime}(z)-q z^{q-1} f^{\prime}(z)-q(q-1) z^{q-2} f(z)=0$.

Recall [9, Theorem 2.1], according to which there cannot be even one ray on which $B(z)$ would be stronger than $A(z)$ in the sense of the Phragmén-Lindelöf indicator, for otherwise all solutions of (1.3) are of infinite order. This happens, for example, when $A(z)$ and $B(z)$ are dual to each other.

Example 5.1 One may observe the necessity of the assumption on the duality of $f$ and $A(z)$ as well as that on the dominance of $A(z)$ over $B(z)$ by the following example: The function $f(z)=\left(e^{z}+e^{-z}\right) e^{z^{q}}, q \in \mathbb{N}$, satisfies

$$
\begin{aligned}
f^{\prime \prime} & +\left\{H(z) e^{z}+H(z) e^{-z}-2 q z^{q-1}\right\} f^{\prime} \\
& -\left\{\left(q z^{q-1}+1\right) H(z) e^{z}+\left(q z^{q-1}-1\right) H(z) e^{-z}-q^{2} z^{2(q-1)}+q(q-1) z^{q-2}+1\right\} f=0
\end{aligned}
$$

for any entire function $H$. When $q=1$, this becomes

$$
f^{\prime \prime}+\left\{H(z) e^{z}+H(z) e^{-z}-2\right\} f^{\prime}-2 H(z) e^{z} f=0
$$

with $f(z)=e^{2 z}+1$. Thus we may use it in order to observe the duality of $A(z)$ and $B(z)$ by several choices of $H$ such as $H(z)=e^{n z}$ for $n \in \mathbb{Z}$ or $H(z)=e^{i z}$.

Here we note that $f(z)=F(z) e^{z^{q}}$ satisfies $\frac{f^{\prime}}{f}=\frac{F^{\prime}}{F}+q z^{q-1}$ and

$$
\frac{f^{\prime \prime}}{f}=\frac{F^{\prime \prime}}{F}+2 q z^{q-1} \frac{f^{\prime}}{f}+\left(q(q-1) z^{q-2}-q^{2} z^{2(q-1)}\right)
$$


so that there is no large freedom to choose the function $F$. For example, taking an Airy function as $F$, we cannot have our desired equation $f^{\prime \prime}+A(z) f^{\prime}+B(z) f=0$ with the exponential polynomial coefficients $A(z)$ and $B(z)$.

Acknowledgements. Ishizaki was supported by JSPS KAKENHI Grant Number 20K03658. Wen was supported by the National Natural Science Foundation of China (No. 11971288 and No. 11771090) and Shantou University SRFT (NTF18029).

\section{References}

[1] Amemiya I. and M. Ozawa, Non-existence of finite order solutions of $w^{\prime \prime}+e^{-z} w^{\prime}+Q(z) w=0$. Hokkaido Math. J. 10 (1981), Special Issue, 1-17.

[2] Frei M., Über die subnormalen Lösungen der Differentialgleichung $w^{\prime \prime}+e^{-z} w^{\prime}+$ konst $\cdot w=0$. Comment. Math. Helv. 36 (1961), 1-8. (German)

[3] Gross F., Factorization of Meromorphic Functions. Mathematics Research Center, Naval Research Laboratory, Washington, D. C., 1972.

[4] Gundersen G. G., On the question of whether $f^{\prime \prime}+e^{-z} f^{\prime}+B(z) f=0$ can admit a solution $f \not \equiv 0$ of finite order. Proc. Roy. Soc. Edinburgh Sect. A 102 (1986), no. $1-2,9-17$.

[5] Gundersen G. G., Finite order solutions of second order linear differential equations. Trans. Amer. Math. Soc. 305 (1988), no. 1, 415-429.

[6] Gundersen G. G., Estimates for the logarithmic derivative of a meromorphic function, plus similar estimates. J. London Math. Soc. (2) 37 (1988), no. 1, 88-104.

[7] Gundersen G. G., E. Steinbart and S. Wang, The possible orders of solutions of linear differential equations with polynomial coefficients. Trans. Amer. Math. Soc. 350 (1998), no. 3, 1225-1247.

[8] Heittokangas J., K. Ishizaki, K. Tohge and Z.-T. Wen, Zero distribution and division results for exponential polynomials. Israel J. Math. 227 (2018), no. 1, 397-421.

[9] Heittokangas J., I. Laine, K. Tohge and Z.-T. Wen, Completely regular growth solutions of second order complex linear differential equations. Ann. Acad. Sci. Fenn. Math. 40 (2015), no. 2, 985-1003.

[10] Heittokangas J. and Z.-T. Wen, Generalization of Pólya's zero distribution theory for exponential polynomials, and sharp results for asymptotic growth. Comput. Methods Funct. Theory, online publ., https://doi.org/10.1007/s40315-020-00336-7, 26 pp.

[11] Langer R. E., On the zeros of exponential sums and integrals. Bull. Amer. Math. Soc. 37 (1931), no. 4, 213-239.

[12] Langley J. K., On complex oscillation and a problem of Ozawa. Kodai Math. J. 9 (1986), no. 3, 430-439.

[13] Moreno C. J., The zeros of exponential polynomials. I. Compositio Math. 26 (1973), $69-78$.

[14] Nevanlinna R., Analytic Functions. Translated from the second German edition by Phillip Emig. Die Grundlehren der mathematischen Wissenschaften, Band 162 Springer-Verlag, New York-Berlin, 1970. 
[15] Ozawa M., On a solution of $w^{\prime \prime}+e^{-z} w^{\prime}+(a z+b) w=0$. Kodai Math. J. 3 (1980), no. 2, 295-309.

[16] Steinmetz N., Zur Wertverteilung von Exponentialpolynomen. Manuscripta Math. 26 (1978/79), no. 1-2, 155-167. (German)

[17] Steinmetz N., Zur Wertverteilung der Quotienten von Exponentialpolynomen. Arch. Math. (Basel) 35 (1980), no. 5, 461-470. (German)

[18] Wen Z. T., G. G. Gundersen and J. Heittokangas, Dual exponential polynomials and linear differential equations. J. Differential Equations 264 (2018), no. 1, 98-114.

\section{J. Heittokangas}

University of Eastern Finland, Department of Physics and Mathematics, P.O. Box 111, 80101 Joensuu, Finland

email: janne.heittokangas@uef.fi

K. Ishizaki

The Open University of Japan, Faculty of Liberal Arts, Mihama-ku, Chiba, JAPAN

email:ishizaki@ouj.ac.jp

K. Tohge

Kanazawa University, College of Science and Engineering, Kakuma-machi, KANAZAWA 920-1192, JAPAN

email:tohge@se.kanazawa-u.ac.jp

Z.-T. Wen

Shantou University, Department of Mathematics, Daxue Road No. 243, Shantou 515063, ChinA

e-mail:zhtwen@stu.edu.cn 